\title{
Neuropathy After Herniorrhaphy: Indication for Surgical Treatment and Outcome
}

\author{
Henri Vuilleumier • Martin Hübner • \\ Nicolas Demartines
}

Published online: 21 January 2009

(C) Société Internationale de Chirurgie 2009

\begin{abstract}
Background Chronic neuropathy after hernia repair is a neglected problem as very few patients are referred for surgical treatment. The aim of the present study was to assess the outcome of standardized surgical revision for neuropathic pain after hernia repair.

Methods In a prospective cohort study we evaluated all patients admitted to our tertiary referral center for surgical treatment of persistent neuropathic pain after primary herniorrhaphy between 2001 and 2006. Diagnosis of neuropathic pain was based on clinical findings and a positive Tinel's sign. Postoperative pain was evaluated by a visual analogue scale (VAS) and a pain questionnaire up to 12 months after revision surgery.

Results Forty-three consecutive patients (39 male, median age 35 years) underwent surgical revision, mesh removal, and radical neurectomy. The median operative time was 58 min (range: 45-95 min). Histological examination revealed nerve entrapment, complete transection, or traumatic neuroma in all patients. The ilioinguinal nerve was affected in 35 patients $(81 \%)$; the iliohypogastric nerve, in 10 patients (23\%). Overall pain (median VAS) decreased permanently after surgery within a follow-up period of 12 months (preoperative 74 [range: 53-87] months versus 0 [range: $0-34$ ] months; $p<0.0001$ ).

Conclusions The results of this cohort study suggest that surgical mesh removal with ilioinguinal and iliohypogastric
\end{abstract}

H. Vuilleumier $(\square) \cdot$ M. Hübner $\cdot N$. Demartines Department of Visceral Surgery, Centre Hospitalier Universtaire Vaudois (CHUV), University Hospital, Lausanne, Rue du Bugnon 46, 1011 Lausanne, Switzerland

e-mail: henri.vuilleumier@chuv.ch neurectomy is a successful treatment in patients with neuropathic pain after hernia repair.

\section{Introduction}

With an incidence of about $10 \%$, chronic inguinal pain after hernia repair remains an important problem with a high socioeconomic impact [1-4]. The number of patients with chronic pain after hernia repair is still underestimated as only a minority are referred for further therapy $[1,2,4]$. Onset of posthernia repair pain usually occurs immediately after surgery [1]. There is little correlation between the type of primary hernia repair and subsequent development of chronic pain, whereas repair of recurrent hernia is an established risk factor [3-7]. Further predictive factors are preoperative pain, severe early postoperative pain, younger age, psychological vulnerability or psychiatric disorder, and workers' compensation $[1,3,4,8]$. Careful clinical and neurological evaluation is crucial for an adequate diagnosis and the ability to offer optimal therapy $[4,9,10]$.

There are two predominant types of posthernia repair pain: nociceptive pain, due predominantly to inflammation, and neuropathic pain, due to nerve injury. The most common type of postoperative pain is a nociceptive pain presenting with tenderness along the inguinal ligament and radiation to the scrotum and the thigh [4]. Nociceptive pain can be somatic or visceral in nature. Somatic pain after hernia repair is mostly due to tissue damage by sutures or mesh fixation devices. The suggested underlying pathomechanism is chronic inflammation resulting from tissue injury that persists in a vicious cycle. Common descriptors of nociceptive pain are "tender," "gnawing," or "pounding" $[1,4,8]$. Neuropathic pain is 
the second most frequent entity, and it is typically a result of surgical injury to a specific nerve (or nerves) [4]. Unlike nociceptive pain, it is amenable to surgical treatment. The nerves involved are either the ilioinguinal, the iliohypogastric, or the genital branch of the genitofemoral nerve. Nerve injury may be caused by partial or complete nerve sectioning, entrapment by sutures and mesh fixation devices, or unintended trauma (e.g., contusion, electrocautery). Secondary trauma involves irritation and compression by foreign material and scar tissue. Neuropathic pain is characterized by a transient electrical stabbing or burning pain that occurs either spontaneously or after a provocation test $[1,3,4,11]$.

Nonsurgical treatment modalities include medical treatment, injection of local anesthetics with or without steroids, cryotherapy, and behavioral therapy [1-3]. Alcohol or phenol injections can be effective in cases of nociceptive pain only [2, 3]. The outcome of patients operated for nociceptive pain is rather disappointing [1, 2, 4 , 9]. In patients with neuropathic pain however, success rates of up to $80 \%$ have been found after surgical revision with radical neurectomy [9-11]. These findings are mainly based on small and heterogeneous patient collectives.

The principal aim of the present study was to prospectively evaluate the success of our standardized technique of nerve resection for the treatment of neuropathic pain occurring after inguinal hernia repair.

\section{Patients and methods}

Between 2001 and 2006, all patients referred to our tertiary center for neuropathic pain after primary groin hernia repair were included in this cohort study. Patients with clinical presentation of nociceptive pain (Table 1) were excluded [4, 8]. Data on patient demographics, primary hernia repair, and pain characteristics were

Table 1 Characteristics of nociceptive and neuropathic pain

\begin{tabular}{lll}
\hline & Nociceptive & Neuropathic \\
\hline Etiology & Tissue damage & Nerve injury \\
Localization & Groin area & Trigger point \\
Duration & Constant & Episodic \\
Aggravating & Strenuous exercise & Walking, sitting \\
Quality & Tender, gnawing, pounding & Stabbing, burning, aching \\
Allodynia & - & + \\
Tinel's sign & - & + \\
\hline
\end{tabular}

Allodynia is pain sensation caused by a normally nonpainful stimulus. Tinel's sign is a clinical test for nerve irritation: light tapping over the area of point tenderness elicits a sensation of tingling or pins-andneedles prospectively assessed. Regular follow-up was scheduled up to 12 months after revision surgery.

Pain: definition, classification, assessment

Pain was defined as chronic when lasting for 3 months or more [1, 4]. Diagnostic features for clinical classification of neuropathic pain are given in Table $1[4,8]$. Tinel's sign was obtained by lightly tapping over the nerve to elicit a sensation of tingling or pins-and-needles over the area of point tenderness. Pain was classified as neuropathic only if the pertinent clinical signs (Table 1) and a positive Tinel's sign were present. Pain was measured on a visual analogue scale (VAS) from 0 (no pain) to 100 (most severe pain). A score of less than 10 was graded as mild pain, a score between 10 and 50 as moderate pain, and a score greater than 50 as severe pain $[12,13]$. A short form of the validated McGill Pain Questionnaire (SF-MPQ) was used for qualitative and quantitative pain assessment [14].

\section{Surgery}

Surgical revision was performed by a single surgeon (H.V.) in a standardized manner [10]. The procedure consisted of removal of the involved mesh or mesh fixation devices and a radical neurectomy of the ilioinguinal and iliohypogastric nerves.

All patients were explored by an anterior approach under epidural or general anesthesia. Dissection was started laterally to the internal inguinal ring in order to identify the ilioinguinal and iliohypogastric nerves in an untouched area where those nerves enter the groin area (Fig. 1). After previous anterior mesh repair, any prosthetic material along the course of the involved nerve was resected systematically. Radical neurectomy consisted of wide proximal and distal sharp transection (Fig. 1) and 5.0 polypropylene ligation of the nerve ends (Prolene, Ethicon, Sommerville, NJ). To prevent neuroma formation, the proximal ends of the ilioinguinal and iliohypogastric nerves were buried within the internal oblique muscle [10]. The genital branch of the genitofemoral nerve was not routinely dissected. After mesh removal, a lightweight mesh (Ultrapro, Ethicon, Somerville, NJ) was placed in the preperitoneal space according to method of Rives [15] in order to reinforce the inguinal floor. All resected nerves were histologically analyzed and the type of nerve lesion was documented.

Statistical analysis

Descriptive statistics are expressed as median (range). Continuous variables between the two groups were compared using the Mann-Whitney U-test. A $p$ value of less than 0.05 was considered as statistically significant. 


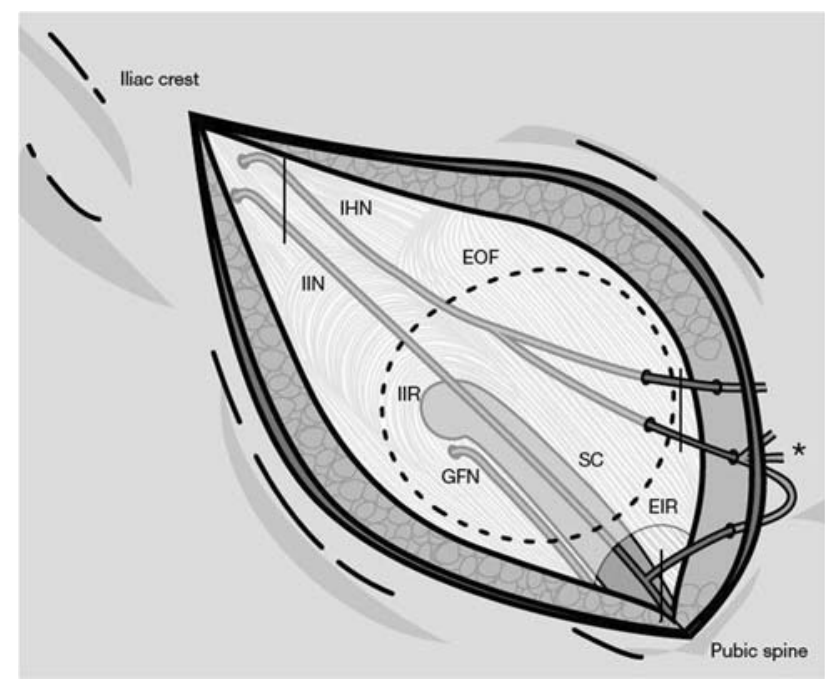

Fig. 1 Concept of radical neurectomy. The iliohypogastric (IHN) and the ilioinguinal nerves (IIN) perforate the posterior part of the transverse abdominal muscle and the internal oblique muscle near the iliac crest. The IHN follows the internal oblique muscle and pierces the external oblique fascia (EOF) above the external inguinal ring (EIR). The IIN accompanies the spermatic cord (SC) and the genital branch of the genitofemoral nerve (GFN) through the EIR. Variable subcutaneous nerve intercommunications $(*)$ are frequent. Most nerve lesions and pronounced adhesions in the present series were encountered in the territory of the primary operation (dotted area). Therefore, dissection was started laterally to the internal inguinal ring (IIR) in order to identify the IHN and IIN in an untouched area close to the iliac crest. Any prosthetic material along the course of the involved nerves was systematically resected (dotted area). Radical neurectomy consisted in wide proximal and distal sharp transection (dashed line)

\section{Results}

\section{Patients}

During the study period, 49 consecutive patients were admitted to our tertiary referral center for surgical treatment of chronic neuropathic pain after primary hernia repair. All patients had previously undergone unsuccessful conservative treatment, consisting of systemic analgesics, injections of local anesthetics and steroids, and physiotherapy. None of the patients had a recurrent hernia at admission. Six patients were excluded for a clear clinical presentation of nociceptive pain (Table 1). The remaining 43 patients (39 males) with neuropathic pain had a median age of 35 years (range: 18-76 years). Patient characteristics and preoperative pain scores related to the primary hernia repair are displayed in Table 2.

\section{Pain}

The onset of pain occurred with a median delay of 18 days (range: 0-28 days) after primary hernia repair. Patients were admitted for surgical revision after a median of 12 months (range: 9-4 months) and a median work incapacity of 6 months (range: $2-14$ months). The median pain score (VAS) was 74 (range: 53-87) and was therefore classified as severe. Description of the pain ranged from burning in $23 / 43(53 \%)$ or stabbing in $16 / 43(37 \%)$ to electric sensation over the inguinal area in $7 / 43$ patients (16\%).

\section{Operative findings}

The median operative time was 58 min (range: 45$95 \mathrm{~min})$. The resected nerves and the type of nerve lesion were confirmed by histopathological examination. All 43 patients had nerve entrapment, complete nerve transection, or traumatic neuroma. Overall, the ilioinguinal nerve was affected in 35 patients $(81 \%)$ and in all patients with previous mesh-plug $(n=6)$ or totally extraperitoneal repair $(n=12)$. The iliohypogastric nerve was damaged in 10 patients overall (23\%), but in 7 of 15 patients with a previous anterior tension-free mesh repair (Table 2). The genital branch of the genitofemoral nerve was not routinely dissected and we found no grossly evident lesion of this nerve in any of our patients.

The type of nerve injury depended on the primary operation. Pain after previous tissue-to-tissue repair was observed in all patients to be due to entrapment of the ilioinguinal and or the iliohypogastric nerve at the inguinal floor suture line. Mesh-plug or tension-free mesh repair resulted in nerve entrapment either by the suture or by the mesh itself, dislocation or incomplete transection of the nerve. In all patients with a previous totally extraperitoneal

Table 2 Patient characteristics and operative findings according to type of primary hernia repair

\begin{tabular}{lllll}
\hline & Tissue-tissue $(n=10)$ & Mesh plug $(n=6)$ & Mesh $(n=15)$ & $15 / 0$ \\
\hline Gender (M/F) & $8 / 2$ & $6 / 0$ & $34(19-76)$ & $10 / 2$ \\
Median age, years (range) & $25(18-54)$ & $50(35-68)$ & $74(56-81)$ & $36(18-54)$ \\
Median pain score, VAS (range) & $71(55-86)$ & 6 & 8 & $80(67-87)$ \\
No. of IIN injuries & 9 & 0 & 7 & 2 \\
No. of IHN injuries & 2 & & $72-84)$ & \\
\hline
\end{tabular}

TEP totally extraperitoneal repair; VAS visual analogue scale (0-100); IIN ilioinguinal nerve; IHN iliohypogastric nerve 
repair, the ilioinguinal nerve was found to be entrapped by mesh fixation devices.

Postoperative outcome

The median pain score (VAS) decreased significantly after surgery (preoperative 74 [range: 53-87] versus 0 [range: $0-$ $34], p<0.0001)$, with 41 patients $(95 \%)$ experiencing complete pain relief (VAS score of 0 ). The two remaining patients noted only partial improvement (VAS: preoperative 67 and 71 versus postoperative 31 and 34 , respectively). All patients made clear distinctions between postrevision incisional pain and preoperative neuropathic pain. The neuropathic component of pain was mostly relieved at the first postoperative examination one week after surgery. The hospital stay was one day in 39 patients and 2 days in four patients. One patient developed a recurrent hernia 9 months after revision and underwent successful total extraperitoneal repair. We observed no testicular atrophy, wound infection, or other related complications during postoperative follow-up periods of 6 (100\%) and 12 months (91\%). All 43 patients returned to work within 6 weeks after operation.

\section{Discussion}

The present study shows that surgical revision with radical neurectomy is a highly effective treatment for chronic neuropathic pain after hernia repair. While the patients in our study were referred for surgical treatment late after onset of symptoms and after a long period of work incapacity (6 months), all of them returned to work within 6 weeks after revision surgery. Therefore, referral to a specialized surgeon should be considered early in patients with refractory neuropathic pain. Our success rate, with full recovery in $100 \%$ and complete pain relief in $95 \%$, compares favorably with published success rates of up to $80 \%$ [9-11]. Critical to success is very careful patient selection, whereby those with nociceptive pain are excluded. Again, the indication for surgery in the present study is the presence of neuropathic pain, based on clinical findings and on a compulsory positive Tinel's sign (Table1).

The accuracy of preoperative clinical diagnosis was confirmed postoperatively by histological examination. All patients had histologically verified nerve injuries. Therefore, in our series, radical neurectomy treated the specific causative problem and resulted in immediate postoperative relief of the neuropathic pain. Because both the ilioinguinal nerve and the iliohypogastric nerve were commonly affected, both should be resected. It follows from these findings, that to prevent neuropathic pain after inguinal hernia repair, the ilioinguinal and the iliohypogastric nerves should be routinely identified during an anterior hernia repair. It is not clear, however, whether these nerves should be prophylactically resected during a primary hernia repair [16-18].

These results support the concept that radical neurectomy is the key point of revision surgery for neuropathic pain. We therefore recommend starting dissection at a point proximal to the internal inguinal ring in order to identify the ilioinguinal and iliohypogastric nerves in an untouched area (Fig. 1). This is of importance as the nerves can be difficult to identify where they are entrapped in the scar tissue or in the mesh prosthesis. The entire length of the potentially entrapped nerves should be resected far proximally and distally [9, 10] (Fig. 1). Because nerve intercommunication is possible between the ilioinguinal and iliohypogastric nerves, both should be radically resected. To prevent nerve scarring within the operative field, the proximal transected nerve ends should be ligated and buried within the fibers of the internal oblique muscle [1, 9-11].

What about the genital branch of the genitofemoral nerve? This tiny branch enters the inguinal canal at the deep inguinal ring and runs adjacent to the external spermatic vessels. Irritation of this nerve typically causes testicular pain, but neurectomy of this branch can cause spermatic blood vessel injury and is generally not recommended [10]. None of our patients had symptoms consistent with injury to the genital branch of the genitofemoral nerve and no intraoperative findings to suggest such injury. Therefore, based on our findings and on the findings of others [9, 1618], we do not advocate triple neurectomy as a standard procedure as recommended by others [10].

We conclude that revision surgery is a successful treatment in patients with neuropathic pain after hernia repair, and we advocate surgical mesh removal and radical neurectomy of the ilioinguinal and iliohypogastric nerves. Neurectomy of the genital branch of the genitofemoral nerve appears to be unnecessary. As specific expertise is required for the optimal treatment of refractory neuropathic pain after hernia repair, patients with this type of pain should be referred to a surgeon who specializes in treating this problem.

Acknowledgments The authors thank Rachel Rosenthal for editorial advice and Marc Grand D'Hautville for providing Fig. 1.

\section{References}

1. Aasvang E, Kehlet H (2005) Chronic postoperative pain: the case of inguinal herniorrhaphy. Br J Anaesth 95:69-76

2. Hindmarsh AC, Cheong E, Lewis MP et al (2003) Attendance at a pain clinic with severe chronic pain after open and laparoscopic inguinal hernia repairs. Br J Surg 90:1152-1154 
3. O'Dwyer PJ, Alani A, McConnachie A (2005) Groin hernia repair: postherniorrhaphy pain. World J Surg 29:1062-1065

4. Poobalan AS, Bruce J, Smith WC et al (2003) A review of chronic pain after inguinal herniorrhaphy. Clin J Pain 19:48-54

5. O'Dwyer PJ, Kingsnorth AN, Molloy RG et al (2005) Randomized clinical trial assessing impact of a lightweight or heavyweight mesh on chronic pain after inguinal hernia repair. $\mathrm{Br}$ J Surg 92:166-170

6. Bay-Nielsen M, Nilsson E, Nordin P et al (2004) Chronic pain after open mesh and sutured repair of indirect inguinal hernia in young males. Br J Surg 91:1372-1376

7. Nienhuijs S, Staal E, Keemers-Gels M et al (2007) Pain after open preperitoneal repair versus Lichtenstein repair: a randomized trial. World J Surg 31:17517

8. Nienhuijs SW, Boelens OB, Strobbe LJ (2005) Pain after anterior mesh hernia repair. J Am Coll Surg 200:885-889

9. Aasvang E, Kehlet H (2005) Surgical management of chronic pain after inguinal hernia repair. Br J Surg 92:795-801

10. Amid PK (2002) A 1-stage surgical treatment for postherniorrhaphy neuropathic pain: triple neurectomy and proximal end implantation without mobilization of the cord. Arch Surg 137:100-104
11. Heise CP, Starling JR (1998) Mesh inguinodynia: a new clinical syndrome after inguinal herniorrhaphy? J Am Coll Surg 187:514-518

12. Collins SL, Moore RA, McQuay HJ (1997) The visual analogue pain intensity scale: what is moderate pain in millimetres? Pain 72:95-97

13. McCarthy M Jr, Chang CH, Pickard AS et al (2005) Visual analog scales for assessing surgical pain. J Am Coll Surg 201:245-252

14. Melzack R (1987) The short-form McGill Pain Questionnaire. Pain 30:191-197

15. Amid PK (2005) Groin hernia repair: open techniques. World J Surg 29:1046-1051

16. Mui WL, Ng CS, Fung TM et al (2006) Prophylactic ilioinguinal neurectomy in open inguinal hernia repair: a double-blind randomized controlled trial. Ann Surg 244:27-33

17. Alfieri S, Rotondi F, Di Giorgio A et al (2006) Influence of preservation versus division of ilioinguinal, iliohypogastric, and genital nerves during open mesh herniorrhaphy: prospective multicentric study of chronic pain. Ann Surg 243:553-558

18. Wijsmuller AR, van Veen RN, Bosch JL et al (2007) Nerve management during open hernia repair. Br J Surg 94:17-22 